\title{
Prediction of Clinical Results with the First Thoracic CT Findings in COVID-2019 Patients; Survey Study
}

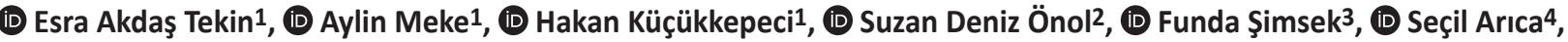 \\ (D) Namigar Turgut ${ }^{1}$ \\ 1 University of Health Seciences Turkey, Prof. Dr. Cemil Taşcıoğlu City Hospital, Clinic of Anesthesiology and Reanimation, İstanbul, Turkey \\ 2 University of Health Seciences Turkey, Prof. Dr. Cemil Taşcıoğlu City Hospital, Clinic of Radiology, Istanbul, Turkey \\ 3 University of Health Seciences Turkey, Prof. Dr. Cemil Taşcıoğlu City Hospital, Clinic of Infectious Diseases and Departmental Microbiology, \\ istanbul, Turkey \\ 4University of Health Seciences Turkey, Prof. Dr. Cemil Taşcıoğlu City Hospital, Clinic of Family Practice, Istanbul, Turkey
}

\section{Abstract}

Objective: This study aimed to investigate the clinical and computed tomography (CT) features associated with severe and critical coronavirus disease-2019 (COVID-19) pneumonia.

Methods: A total of 50 patients with critical COVID-19 pneumonia, receiving mechanical ventilation support in the intensive care unit (ICU) due to moderate-severe acute respiratory distress syndrome (ARDS), were included in this retrospective study. Chest CT, laboratory results, and clinical conditions of patients were concurrently identified. Only the first chest CT findings of patients were reviewed by 50 physicians and they were asked about their opinions on the laboratory results and clinical conditions of patients. Data were then compared with the real-time data. The efficacy of the first chest CT findings in diagnosis and treatment guidance was analyzed.

Results: The simultaneous laboratory analyses of patients receiving mechanical ventilation support due to moderate-severe ARDS found a C-reactive protein of $>40 \mathrm{mg} / \mathrm{L}$ in $72 \%$, D-dimer was $>1000 \mathrm{ng} / \mathrm{mL}$ and ferritin was $>500 \mathrm{ng} / \mathrm{mL}$ in $57 \%$, and lymphocyte count $<800 \mu \mathrm{L}$ in $50 \%$ of patients. While $53 \%$ of participants proposed that patients would be treated in the ICU, $28.2 \%$ suggested the patients be treated in inpatient care. A total of $60.2 \%$ of participants stated that the patients suffered from moderate-severe ARDS based on CT findings.

Conclusion: A correlation was found between the clinical symptoms, laboratory analyses, and CT findings of patients presenting with severe COVID-19. The first chest CT alone may help a physician to determine the disease severity; however, additional laboratory data and clinical follow-up are needed to evaluate the prognosis and plan the treatment, as many factors are involved in determining the disease severity.

Keywords: COVID-19, SARS-CoV-2, ARDS, computed tomography

\section{INTRODUCTION}

Coronavirus disease-2019 (COVID-19) infection, a highly contagious disease caused by the severe acute respiratory syndrome coronavirus-2 virus, was first reported in Wuhan, China, and the World Health Organization declared this increasingly spreading disease as a global public health emergency (pandemic) on January 30, 2020 (1). Chest computed tomography (CT) plays an important role in the diagnosis and treatment of COVID-19. The common chest CT findings of COVID-19 are multiple groundglass opacity, consolidation, and interlobular septal thickening in both lungs, which are mostly distributed under the pleura. A significant correlation between the severity of the pulmonary inflammation, basic clinical symptoms, and laboratory test results was reported (2,3). In addition, data on the efficacy of chest $\mathrm{CT}$ findings alone in predicting the clinical disease 
prognosis is limited (4). Thus, this study aimed to investigate the validity of the first CT findings in treatment guidance, beyond specific diagnosis, by the physicians involved in the diagnosis and treatment of patients with COVID-19.

\section{METHODS}

The study was approved by the Scientific Research Platform of Directorate General for Health Services, the Ministry of Health of the Republic of Turkey, and the Ethics Committee of University of Health Seciences Turkey, Prof. Dr. Cemil Tascioglu City Hospital, (no: 48670771-514.10, 12.05.2020/158). A total of 50 out of 320 patients, admitted to the intensive care unit (ICU) due to COVID-19 [severe COVID-19-related acute respiratory distress syndrome (ARDS)] between March 20, 2020, and June 1, 2020, were included in the study through random selection.

The first chest CT scans of patients, taken before the admission to the ICU, were blindly evaluated by 50 physicians, without receiving information, including the names and recent clinical conditions of patients. Afterward, three questions were asked to all participants and the answers were noted. The actual data of 50 patients were compared to the given answers.

The three questions :

1. "Where should this patient be followed up and treated according to the examined thoracic tomography data?"
a. Quarantine at home
b. Treatment in the hospital clinic
c. In intensive cared. No necessary treatment

2. "Are there signs of severe ARDS in the examined thoracic tomography?"

a. Yes, partial pressure of oxygen/fraction of inspired oxygen $\left(\mathrm{PaO}_{2} / \mathrm{FiO}_{2}\right)$ of $<100 \mathrm{mmHg}$, indication severe ARDS

b. Yes, $\mathrm{PaO}_{2} / \mathrm{FiO}_{2}$ of $100-200 \mathrm{mmHg}$, indication moderate ARDS

c. Yes, $\mathrm{PaO}_{2} / \mathrm{FiO}_{2}$ of $200-300 \mathrm{mmHg}$, indication mild ARDS

d. No, $\mathrm{PaO}_{2} / \mathrm{FiO}_{2}$ of $>300$

3. "What kind of treatment plan is suitable if the patient is followed up in the ICU according to the examined thoracic tomography data?"

a. Medical treatment + supportive treatment + invasive mechanical ventilation

b. Medical treatment + supportive treatment + non-invasive mechanical ventilation (HFNC)

\section{Statistical Analysis}

Data were expressed as mean or median, and answers were calculated in percentage (\%).

\section{Inclusion and Exclusion Criteria}

Over 18 years old, with COVID-19- related severe ARDS diagnosis in the ICU $\left(\mathrm{PaO}_{2} / \mathrm{FiO}_{2}\right.$ of $\left.<100 \mathrm{mmHg}\right)$

\section{RESULTS}

All 50 physicians answered 7,500 questions in 2,500 surveys. Three CT scanners (Scanner-Toshiba-Alexion 16 slice, Scanner Philips-Brilliance 40 -slice, ans Scanner Philips-Ingenuity Core 128-slice) were used for examinations. The simultaneous laboratory analysis found a C-reactive protein (CRP) of $>40 \mathrm{mg} / \mathrm{L}$ in $72 \%$ [minimum-maximum (min-max) (median) 3.24-513.78 (86.99)]; D-dimer was $>1000 \mathrm{ng} / \mathrm{mL}$ [min-max (median) 7827.000 (1100)] and ferritin was $>500 \mathrm{ng} / \mathrm{mL}$ [min-max (median) 24.5-8378 (614.6)] in 57\%; and lymphocyte count of $<800 \mu \mathrm{L}$ [min-max (median) $0.2-16.15$ (0.92)] in 50\% of patients (Table 1, Figure 1a-d).

To the question "Where should this patient be followed up and treated according to the examined thoracic tomography data?", $53 \%$ of participants responded as "in the ICU", $28.2 \%$ as "in the inpatient care", $17.4 \%$ as "in quarantine at home", and $1.4 \%$ stated that no treatment was required (Figure 2).

To the question "Are there signs of severe ARDS in the examined thoracic tomography?", $60.2 \%$ of participants responded as moderate-severe ARDS, $19.6 \%$ as mild ARDS, and 20.2\% stated that the clinical presentation was not ARDS (Figure 3). All patients included were in the ICU due to severe ARDS.

To the question "What kind of treatment plan is suitable if the patient is followed up in the ICU according to the examined thoracic tomography data?", $58.2 \%$ of participants responded as medical treatment + supportive treatment + HFNC and

\begin{tabular}{|l|l|l|}
\hline \multicolumn{3}{|l|}{ Table 1. COVID-19-associated acute-phase reactants of patients } \\
\hline \multirow{2}{*}{ CRP (mg/L) } & Min-max & $3.24-513.78$ \\
\cline { 2 - 3 } & (Median) & $(86.99)$ \\
\hline \multirow{2}{*}{ Ferritin (ng/mL) } & Min-max & $24.5-8378$ \\
\cline { 2 - 3 } & (Median) & $(614.6)$ \\
\hline \multirow{2}{*}{ D-dimer (ng/mL) } & Min-max & $78-27.000$ \\
\cline { 2 - 3 } & (Median) & $(1100)$ \\
\hline \multirow{2}{*}{ Lymphocyte ( $\mu$ L) } & Min-max & $0.2-16.15$ \\
\cline { 2 - 3 } & (Median) & $(0.92)$ \\
\hline $\begin{array}{l}\text { CoVID-19: Coronavirus disease-2019, Min: Minimum, max: Maximum, CRP: C-reactive } \\
\text { protein }\end{array}$ \\
\hline
\end{tabular}


41.8\% as medical treatment + supportive treatment + invasive mechanical ventilation (Figure 4).

\section{DISCUSSION}

This study analyzed the chest CT findings of 50 patients treated for severe COVID-19-related ARDS in the ICU examined by 50

\section{$\mathrm{CRP}(\mathrm{mg} / \mathrm{l})$}

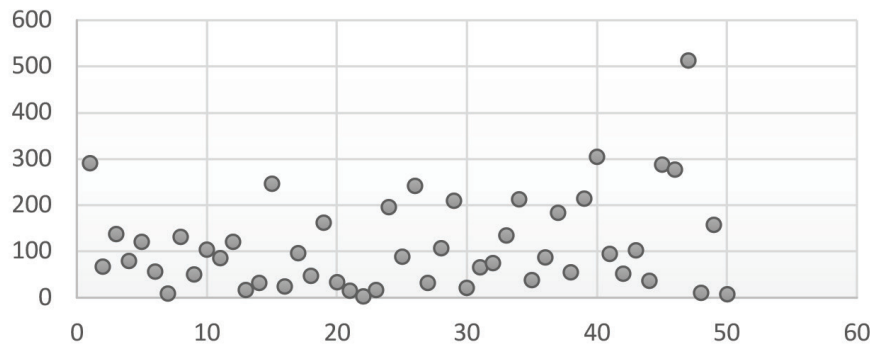

Figure 1a. CRP (mg/L) values of patients CRP: C-reactive protein

\section{D-Dimer (ng/ml)}

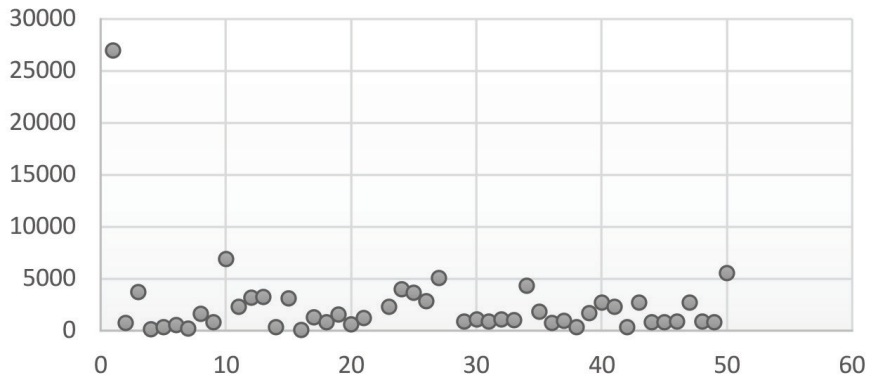

Figure 1b. D-dimer $(\mathrm{ng} / \mathrm{mL})$ values of patients

\section{Ferritin}

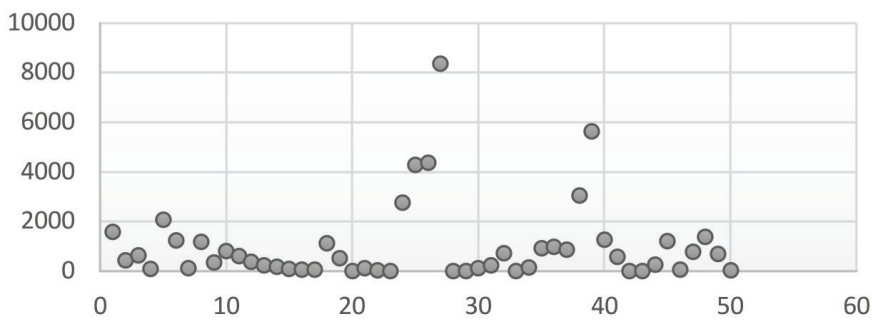

Figure 1c. Ferritin $(\mathrm{ng} / \mathrm{mL})$ values of patients

\section{Lymphocyte count $(\mu \mathrm{l})$}

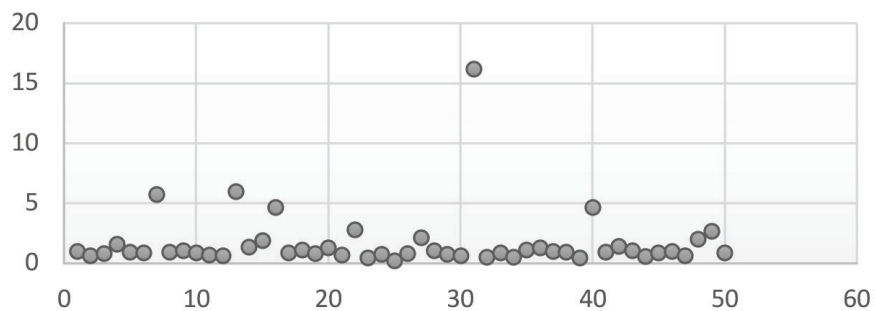

Figure 1d. Lymphocyte $(\mu \mathrm{L})$ values of patients physicians without the real-time laboratory test results, as well as the clinical data and the validity of first CT findings alone in treatment guidance, beyond specific diagnosis.

The meta-analyses of the high-risk group of patients with symptomatic COVID-19 primarily observed the ground-glass opacities (83.31\%) and consolidation involving the bilateral lungs

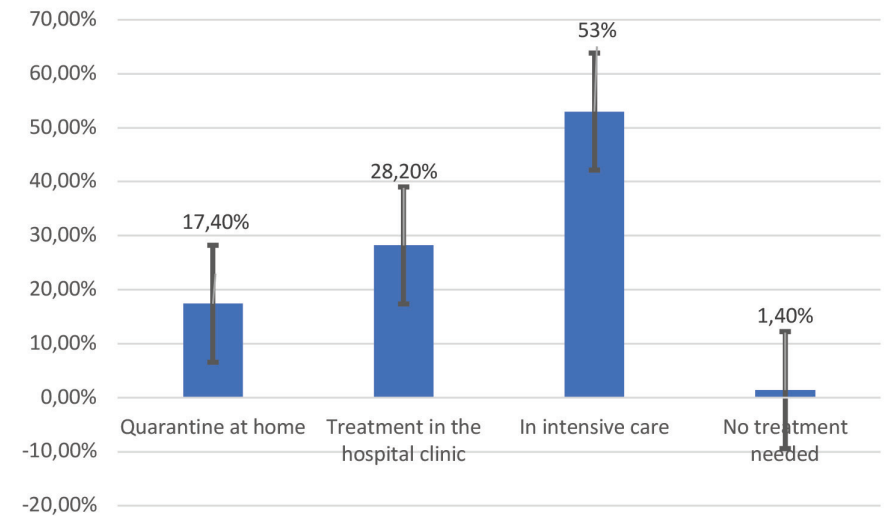

Figure 2. "Where should this patient be followed up and treated according to the examined thoracic tomography data?"

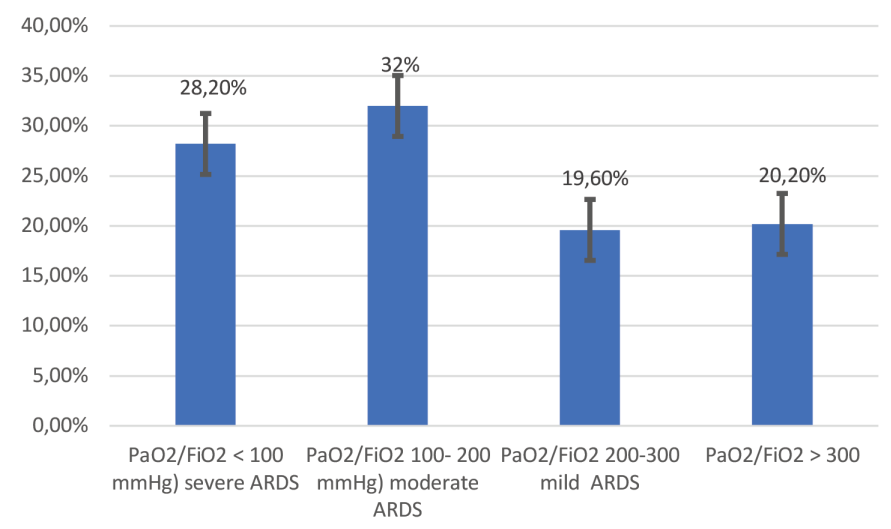

Figure 3. "Are there signs of severe ARDS in the examined thoracic tomography?"

ARDS: Severe acute respiratory distress syndrome

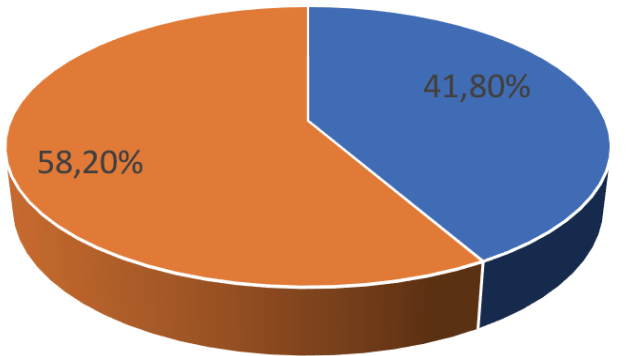

- Medical treatment + Supportive treatment + IMV

- Medical treatment + Supportive treatment +NIMV

Figure 4. "What kind of treatment plan is suitable if the patient is followed up in the intensive care unit according to the examined thoracic tomography data?" 
in a peripheral distribution (76.95\%) in the chest CT. Compared to ordinary patients, characteristics specific to COVID-19 in the thoracic CT play an important role in guiding the treatment and evaluating the disease severity $(5,6)$.

While one of every five physicians who participated in the study diagnosed COVID-19 based on the chest CT, they incorrectly predicted the clinical course and prognosis of the disease based on the first CT findings alone, without clinical findings and laboratory test results. Contrarily, $17.4 \%$ of them suggested quarantine at home.

Advanced age, comorbidities, dyspnea, chest pain, cough, phlegm, decreased lymphocytes, and elevated inflammation indicators are risk factors for COVID-19 pneumonia (6). Significant differences were found between the ordinary patients and patients with severe/critical conditions in terms of clinical symptoms, laboratory examinations, and CT findings. Many factors related to the disease severity may help physicians identify the disease severity and evaluate the prognosis (4). Studies indicated a significant correlation between the severity of the pulmonary inflammation in the CT findings and main clinical symptoms (cough and high fever in $73 \%$ and $76 \%$ of patients, respectively) and laboratory test results in patients confirmed with coronavirus (7). No abnormality was observed in the thoracic CT scans of the early-stage (0-4 days). CT is a useful tool for evaluating the changes in the pulmonary abnormalities of patients in the acute and recovery period (7-9). Therefore, physicians need to evaluate the chest CT of patients in combination with the clinical course and laboratory findings.

Real-time polymerase chain reaction (RT-PCR) is accepted as a reference standard test in COVID-19 diagnosis. Chest CT findings may exist in the early stages before the onset of symptoms, and chest CT sensitivity is quite high, not only in the diagnosis but also in the follow-up of patients with COVID-19 having falsenegative RT-PCR results (10). Our study observed a correlation between the initial CT findings, real-time laboratory test results, and clinical course. While $28.2 \%$ of physicians diagnosed severe ARDS based on the first chest CT scan, only $53 \%$ of them suggested a follow-up in the ICU and $28.2 \%$ suggested a follow-up in the inpatient care. Therefore, $20.2 \%$ of the participants declared that ARDS will not be developed. In reality, 50 patients were treated in the ICU due to respiratory distress with IMV. The arterial blood gas analysis identified a $\mathrm{PaO}_{2} / \mathrm{FiO}_{2}$ of $<100 \mathrm{mmHg}$, indicating severe ARDS.

Hyperinflammation is observed in patients with critical COVID-19, and related biomarkers may be beneficial in risk classification. COVID-19 severity and poor prognosis were found related to $96 \%$ serum CRP, $85 \%$ procalcitonin, $77 \%$ D-dimer, and $76 \%$ serum ferritin in the performed meta-analyses. The severity and the disease prognosis may be evaluated in a patient with positive PCR without CT findings by evaluating the acutephase reactants in the first stage (11). Chest $C T$ is guiding the clinical diagnosis and is effective in predicting the treatment and the prognosis. All 50 patients included in the study were PCR-positive. The examined acute-phase reactants were found proportionate to the CT findings and the severity of hypoxemia. CRP was especially the most elevated acute-phase reactant, with the disease severity, followed by ferritin and D-dimer.

While acute-phase reactants were concurrently found to be as lymphocyte count of $680 \mu \mathrm{L}$, CRP of $90.81 \mathrm{mg} / \mathrm{L}$, ferritin of $637 \mathrm{ng} /$ $\mathrm{mL}$, and D-dimer of $3.720 \mathrm{ng} / \mathrm{mL}$ (Figure 5) in a patient presenting with moderate to severe viral infection symptoms in the chest $\mathrm{CT}$, another acute-phase reactant count was concurrently found to be normal in a patient with severe viral infection symptoms in the chest CT (lymphocyte count of $156 \mu \mathrm{L}$, CRP of $27.6 \mathrm{mg} / \mathrm{L}$, ferritin of $121 \mathrm{ng} / \mathrm{mL}$, and D-dimer $705 \mathrm{ng} / \mathrm{mL}$ ) (Figure 6). Further studies on the correlation between the acute-phase reactants and chest CT findings are required. Treatment in the ICU has become increasingly difficult; therefore, early recognition of severe cases is necessary for a timely triaging of patients. Clinical conditions and concurrent comorbidities determine the necessity of treatment in the ICUs, whereas several laboratory parameters may help evaluate the disease severity. Physicians should also take the low lymphocyte count into account, as well as the CRP, D-dimers, ferritin, cardiac troponin, and interleukin-6 serum

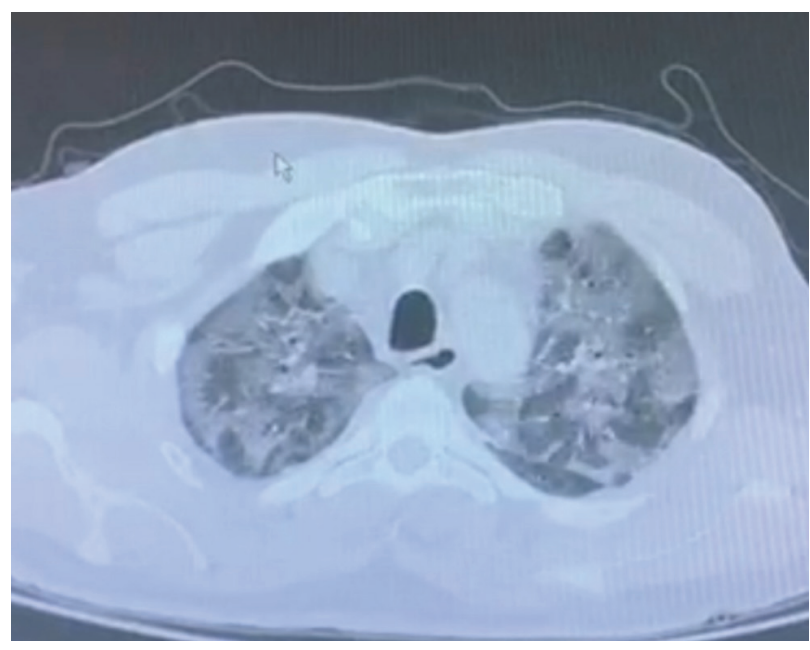

Figure 5. Diffuse ground-glass infiltrations showing moderate to severe parenchymal involvement from the apex to the base, with a tendency to unite in the parenchyma of both lungs, fibrotic changes, and interlobular septal thickening, causing crazy-paving patterns. A pleural fluid of slightly over $1 \mathrm{~cm}$ in width was observed in the bilateral base. Diffuse infiltrates and accompanying pleural fluid suggested moderate to severe viral pneumonia 


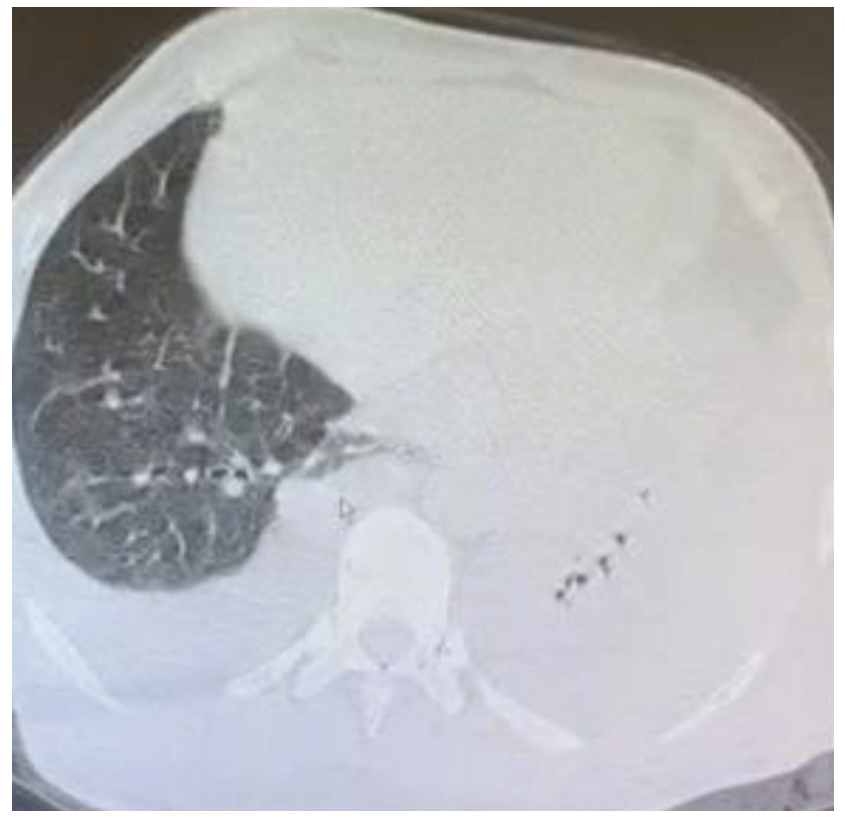

Figure 6. Pleural effusion was observed in the posterior base in both hemothorax. In the lung parenchyma, diffuse infiltration appearances, which were consolidated in the lower lobes of both lung parenchymas and caused the loss in total lung ventilation in the lower lobe of the left lung, were observed. The identified findings were evaluated in favor of severe specific viral infection. Concurrent acute-phase reactants: Lymphocyte count of $156 \mu \mathrm{L}$, CRP of $27.6 \mathrm{mg} / \mathrm{L}$, ferritin of $121 \mathrm{ng} / \mathrm{mL}$, and D-dimer of $705 \mathrm{ng} / \mathrm{mL}$

CRP: C-reactive protein

levels, to determine severe and fatal COVID-19 in inpatient care. The disease course is likely to be negative when one or more of these parameters are altered (12).

\section{CONCLUSION}

In conclusion, a correlation was found between the clinical symptoms, laboratory analyses, and CT findings of patients with severe to critical COVID-19. First chest CT alone may help physicians to determine the severity of the disease; however, additional laboratory data and clinical follow-up are needed to evaluate the prognosis and plan the treatment, as many factors are related to the severity of the disease.

\section{Ethics}

Ethics Committee Approval: The study was approved by the Scientific Research Platform of Directorate General for Health Services, the Ministry of Health of the Republic of Turkey, and the Ethics Committee of University of Health Seciences Turkey, Prof. Dr. Cemil Tascioglu City Hospital, (no: 48670771-514.10, 12.05.2020/158).
Informed Consent: Survey study.

Peer-review: Internally peer-reviewed.

\section{Authorship Contributions}

Concept: E.A.T., A.M., H.K., S.D.Ö., N.T., Design: E.A.T., N.T., Data Collection or Processing: E.A.T., A.M., H.K., Analysis or Interpretation: E.A.T., N.T., Literature Search: E.A.T., N.T., Writing: F.S., S.D.Ö., S.A., N.T.

Conflict of Interest: No conflict of interest was declared by the authors.

Financial Disclosure: The authors declared that this study received no financial support.

\section{REFERENCES}

1. Gorbalenya AE, Baker SC, Baric RS, de Groot R, Drosten C, Gulyaeva AA, et al. Severe acute respiratory syndromerelated coronavirus: the species and its viruses - a statement of the coronavirus study group. BioRxiv 2020;1-15.

2. Ye Z, Zhang Y, Wang Y, Huang Z, Song B. Chest CT manifestations of new coronavirus disease 2019 (COVID-19): a pictorial review. Eur Radiol 2020;30:4381-9.

3. Bai HX, Hsieh B, Xiong Z, Halsey K, Choi JW, Tran TML, et al. Performance of radiologists in differentiating COVID-19 from non-COVID-19 viral pneumonia at chest CT. Radiology 2020;296:E46-E54.

4. Li K, Wu J, Wu F, Guo D, Chen L, Fang Z, et al. The clinical and chest CT features associated with severe and critical COVID-19 pneumonia. Invest Radiol 2020;55:327-31.

5. Bao C, Liu X, Zhang H, Li Y, Liu J. Coronavirus disease 2019 (COVID-19) CT findings: a systematic review and meta-analysis. J Am Coll Radiol 2020;17:701-9

6. Xu X, Yu C, Qu J, Zhang L, Jiang S, Huang D, et al. Imaging and clinical features of patients with 2019 novel coronavirus SARS-CoV-2. Eur J Nucl Med Mol Imaging 2020;47:1275-80.

7. Wu J, Wu X, Zeng W, Guo D, Fang Z, Chen L, et al. Chest CT Findings in patients with coronavirus disease 2019 and its relationship with clinical features. Invest Radiol 2020;55:257-61.

8. Ding X, Xu J, Zhou J, Long Q. Chest CT findings of COVID-19 pneumonia by duration of symptoms. Eur J Radiol 2020;127:109009.

9. Yoon SH, Lee KH, Kim JY, Lee YK, Ko H, Kim KH, et al. Chest Radiographic and CT Findings of the 2019 novel coronavirus disease (COVID-19): analysis of nine patients treated in Korea. Korean J Radiol 2020;21:494500 .

10. Gündüz Y, Öztürk MH, Tomak Y. The usual course of thorax CT findings of COVID-19 infection and when to perform control thorax CT scan. Turk J Med Sci 2020;50:684-6.

11. Huang I, Pranata R, Lim MA, Oehadian A, Alisjahbana B. C-reactive protein, procalcitonin, D-dimer, and ferritin in severe coronavirus disease-2019: a meta-analysis. Ther Adv Respir Dis 2020;14:1753466620937175.

12. Velavan TP, Meyer CG. Mild versus severe COVID-19: laboratory markers. Int J Infect Dis 2020;95:304-7. 